\title{
NATURALEZA Y UNIDAD LÓGICA DE LAS CATEGORÍAS EN DUNS ESCOTO'
}

\author{
HÉCTOR HERNANDO SALINAS \\ Pontificia Universidad Javeriana (Bogotá)
}

\begin{abstract}
RESUMEN: En este trabajo se estudia la primera concepción escotista sobre las categorías y sobre el objeto del tratado aristotélico de las Categorías. El artículo se concentra sobre las tres primeras cuestiones del comentario de Duns Escoto al texto de Aristóteles. Una de las novedades del texto es su tematización del concepto mismo de categoría, entendido como concepto lógico y como objeto propio del tratado aristotélico. Esta aproximación permite a Escoto distinguir el orden lógico y el orden metafísico en el tratamiento de las categorías. En el primero, la lógica se ocupa del concepto de categoría y, en el segundo, la metafísica se ocupa de la diversidad categorial y de su referencia a la categoría de la substancia.
\end{abstract}

PALABRAS CLAVE: Juan Duns Escoto, Aristóteles, categorías, lógica medieval, metafísica medieval.

\section{Duns scotus on the nature and logical unity of the categories}

ABSTRACT: This work studies the first scotist conception of the categories and of the objet of the Aristotelian treatise the Categories. This article focuses on the three first questions of Scotus's commentary to Aristotle's text. One of the most important innovations of the commentary is its treatment of the concept of "category» itself, understood as a logical concept and as proper subjet of the Aristotelian treatise. This approach allows Scotus to distinguish the logical and the metaphysical order in the treatment of categories. In the logical order, this science deals with the concept of category and, the metaphysical order deals with the categorical diversity and its reference to the category of substance.

KEY WORDS: Duns Scotus, Aristotle, categories, medieval logic, medieval metaphysics.

\section{INTRODUCCIÓN}

¿Cuál es el objeto del tratado aristotélico de las Categorías? ¿Cómo se relaciona este objeto con el problema de la multiplicidad categorial? ¿Pertenece el tratado de las Categorías al dominio de la lógica o al de la metafísica? A propósito de estas preguntas, el comentario de Duns Escoto a las Categorías constituye un momento central en la historia de la recepción del texto aristotélico, en la interpretación del estatuto de las categorías y en la historia del desarrollo del pensamiento escotista.

Es ya una tradición consolidada en la historiografía filosófica, presentar a Duns Escoto, no sin razón, como el primer autor que defiende la tesis de la univocidad del ser $^{2}$. Sin embargo, en su obra temprana se manifiesta otro panorama. En la

1 Este artículo es uno de los resultados del proyecto de investigación 006268: Juan Duns Escoto antes de la univocidad del ser, inscrito en la Vicerrectoría de Investigación de la Pontificia Universidad Javeriana, Bogotá.

2 El texto fundamental en que se lleva a cabo este proyecto se encuentra en Juan Duns Escoto, Ordinatio parte I, distinción 3, qq. 1-3 (Vat. III, 1-123). Cfr. La introducción a la traducción francesa de este texto en O. Boulnors "La destruction de l'analogie et l'instauration de la métaphysique», en: Jean Duns Scot, Sur la connaissance de Dieu et l'univocité de l'étant, PUF, París 1988, 11-81. Del mismo autor, también puede consultarse «Analogie et univocité selon Duns Scot: La double 
cuarta cuestión de su comentario a las Categorías $^{3}$, Escoto rechaza sucesivamente la univocidad y la analogía semánticas del término "ser», siguiendo una estrategia que lE conduce a defender su equivocidad lógica ${ }^{4}$. Esta es la primera posición de Escoto, antes de sostener la tesis de la univocidad del ser.

Es por ello que la lectura del comentario, de sus tesis centrales y de los argumentos que las sostienen, permite acceder a la primera solución escotista a la pregunta por el objeto del tratado de las Categorías y al problema de la naturaleza de las categorías. En este punto, no se debe perder de vista que la pregunta por el objeto del tratado descansa sobre la concepción medieval de la ciencia demostrativa derivada de la lectura de los Segundos Analíticos de Aristóteles ${ }^{5}$. Así, al preguntarse por el objeto del tratado, Escoto de hecho se está preguntado por el objeto de indagación científica del que este se ocupa. En el latín medieval, el término para referirse a este objeto es subiectum.

Así pues, antes de demostrar la equivocidad del término «ser», Escoto consagra las primeras tres cuestiones de su comentario a la determinación del objeto del tratado de las Categorías. Esta investigación previa es fundamental para comprender cómo Escoto puede salvaguardar tanto la unidad de la ciencia lógica de las

destruction», en: Les Études philosophiques, «L'analogie», 3-4, 1989, 347-369. Para una presentación de conjunto de los efectos de esta fundación de la metafísica como ciencia trascendental, ver el trabajo de Honnefelder, L., La métaphysique comme science transcendantale: entre le Moyen Âge et les Temps modernes, PUF, París 2002.

3 Duns Scoti, I., Questiones In Librum Porphyrii Isagoge et Quaestiones Super Praedicamenta Aristotelis, ANDREws, R. et al. (eds), en: Opera philosophica I, The Franciscan Institut, St. Bonaventure (NY) 1999, q. 4 Utrum ens sit decem praedicamentis univocum, pp. 273-292. En adelante se cita así: Duns Scotus, In Praed., q. 1 n. 1 (OPh, I, 243), comentario a las Categorías, cuestión 1, parágrafo 1, en las Opera Philosophica de Escoto, volumen I, página 243. En este artículo, salvo mención contraria, todas las traducciones del latín son mías.

4 Duns Scotus, In Praed., q. 4, n. 26 (OPh, I, 280): «Propter hoc dicendum est quod ens non est univocum decem praedicamentis. Tamen quomodo se habeat, dubium est, utrum pure aequivoce vel analogice»; y Duns Scotus, In Praed., q. 4 n. 38 (OPh, I, 285): «Intelligendum tamen quod vox, quae apud logicum simpliciter aequivoca est, quia scilicet aeque primo importat multa, apud metaphysicum vel naturalem, qui non considerant vocem in significando sed ea quae significantur secundum illud quod sunt, est analoga, propter illud quod ea quae significantur, licet non in quantum significantur; tamen in quantum exsistunt habent ordinem inter se. Ideo 'ens' a metaphysico in IV et VII Metaphysicae ponitur analogum ad substantiam et accidens, quia scilicet haec quae significantur, in essendo habent ordinem; sed apud logicum est simpliciter aequivocum, quia in quantum significantur per vocem, aeque primo significantur». La cursiva es mía.

5 Cf. RiJk, L. M. de, «The Posterior Analytics in the Latin West», en: Asztalos, M., Murdoch, J. E. \& Nirniluoto, I. (eds.), Knowledge and the Sciences in Medieval Philosophy. Proceedings of The Eighth International Congress of Medieval Philosophy, Vol. 1, Acta Philosophica Fennica, Helsinki 1990, 104-127. Sobre la ciencia demostrativa ver Serene, E., "Demonstrative science», en Kretzmann, N., Kenny, A., \& Pinborg, J. (eds.), The Cambridge History of Later Medieval Philosophy, Cambridge University Press, Cambridge 1982, 496-517 (y sobre Escoto p. 507-513). Y recientemente, PASNaU, R., «Science and Certainty», en: Pasnau, R. \& Dyke, Ch. van (eds.), The Cambridge History of Medieval Philosophy, vol. I, Cambridge University Press, Cambridge 2010, 357-368, recordaba que «although we now tend to focus on the recovery of Aristotle's natural philosophy, metaphysics, and ethics, it is arguably the Posterior Analytics — not the Ethics, the Metaphysics, the Physics, or the De anima - that had the most pervasive influence on scholastic thought. For it is here that Aristotle sets out the methodological principles that are to be followed in the pursuit of systematic, scientific knowledge: what the Latin tradition would call scientia» (p. 257). 
categorías como la equivocidad del término «ser». Es así que, como lo ha subrayado O. Boulnois, Escoto ofrece aquí una aproximación a la noción de «categoría en general», por contraste con el texto mismo de las Categorías $^{6}$. Esta es la principal novedad de su lectura, pues es con ella que puede garantizar la unidad lógica del tratado de las Categorías cuyo objeto es el concepto intencional de categoría, separándola y distinguiéndola tanto del orden real en el que la diversidad de las categorías se reduce a la sustancia (bajo una unidad de analogía), como también del orden del lenguaje en el que tradicionalmente se situaba el estudio de las categorías.

En este trabajo nos vamos a consagrar a la lectura de estas primeras tres cuestiones del comentario para ver cómo se construye esta reflexión sobre el concepto lógico de "categoría» y las implicaciones que tiene para la ciencia lógica de las categorías y su relación con el lenguaje y la metafísica ${ }^{7}$. La progresión de estas cuestiones es muy clara.

La primera cuestión se pregunta si el libro de las Categorías tiene por objeto las diez voces que significan los diez géneros más generales de cosas. La solución a la pregunta separa el orden de la voz, orden lingüístico en que se expresa el pensamiento, del orden conceptual o intencional donde Escoto sitúa el objeto del que se ocupa el tratado, objeto anterior e indiferente a su expresión en el lenguaje.

La segunda cuestión se pregunta si el objeto del tratado son las diez categorías. La solución distingue dos maneras de tratar las categorías. En el tratado de las $\mathrm{Ca}$ tegorías, ellas se tematizan «en cuanto se les atribuye algo causado por la razón» (q. 2, n. 19). Este es el punto de vista del lógico y es este el punto de vista del tratado. La consideración de las categorías, en tanto lo que ellas son, corresponde por su parte, a la metafísica (q. 2, n. 24). Es pues esa dimensión de lo causado por la razón la que funda el espacio de consideración lógica de las categorías.

La tercera cuestión se pregunta si existe un predicado intencional unívoco a las categorías. En otros términos, si se puede predicar un atributo que en el orden lógico sea unívoco a las categorías. La pregunta supone la solución de la cuestión anterior y se sitúa fuera del orden de preocupación del metafísico. La solución a esta cuestión distingue entre la diversidad real de las categorías y la unidad que el intelecto produce al considerarlas según un único modo de concebir (modus concipiendi). Escoto distingue entre lo concebido por el intelecto y el modo de concebirlo, de tal modo que la unidad intencional se funda sobre el modo de concebir y no sobre lo concebido.

\section{EL TRASFONDO PORFIRIO-BOECIANO Y LA RUPTURA AVICENIANA}

En la tradición medieval, la lectura e interpretación del tratado de las Categorías dependen directamente del modo en que Boecio se planteó el problema del objeto

\footnotetext{
6 Boulnois, O., «Les catégories selon Duns Scot», en: Carbajo NúñEz, M. (ed.), Giovanni Duns Scoto: Studi e ricerche nel VII Centenario della sua morte, Vol.1, Antonianum, Roma 2008, 358.

7 En lo que sigue, utilizo para la versión castellana de esas cuestiones mi reciente traducción: SAlinas, H. H. «Juan Duns Escoto: Cuestiones sobre las Categorías de Aristóteles, qq. 1-3», en: Universitas Philosophica, 67, 2016, 275-302.
} 
del tratado, siguiendo la lectura porfiriana del texto de Aristóteles ${ }^{8}$, así como su propia lectura de la Isagoge. En el mundo latino, desde Boecio se lee la Isagoge como lo que se supone es para Porfirio: una introducción a las Categorías. O. Boulnois ha sostenido que la relación entre las Categorías de Aristóteles y la Isagoge de Porfirio constituye un círculo hermenéutico en la medida en que la respuesta a la pregunta por el estatuto del universal, en el comentario a la Isagoge, dirige una interpretación de las Categorías, del mismo modo que una interpretación de las Categorías (¿son palabras, cosas o conceptos?) interviene sobre las decisiones interpretativas en la lectura de la Isagoge .

En el comentario a la Isagoge, Escoto distingue la naturaleza de lo concebido por el intelecto del modo de concebirlo (modus concipiendi), siendo este último algo propio del intelecto, causado por él. El modo en que el intelecto concibe una naturaleza, no es otro que el modo universal. Esta teoría de origen aviceniano defiende la distinción entre la naturaleza sin más, anterior e indiferente a sus modos de darse en el intelecto y fuera del intelecto. Por ejemplo, en su Logyca, Avicena presenta del siguiente modo, la distinción entre el animal en sí y su modo de darse en el intelecto (por una aprehensión universal) y en la realidad extramental (como naturaleza individual):

Animal es por sí algo, y es el mismo si es sensible o si es un concepto en el alma. En sí, sin embargo, ni es universal, ni es singular. En efecto, si fuera universal, de tal modo que la animalidad en tanto animalidad fuera universal, se seguiría necesariamente, por el contrario que ningún animal sería singular, y todo animal sería universal. Pero si animal en tanto animal fuera singular, sería imposible que hubiera más de un singular, a saber, el mismo singular al cual pertenece la animalidad, y sería imposible que otro singular fuera animal. Además, animal por sí es un cierto concepto en la mente que es animal; y en tanto es inteligido como animal, solo es animal. Pero, si además de esto, se intelige que es universal o singular u otra cosa, entonces se intelige además de esto que es animal, algo a lo que le ocurre la animalidad ${ }^{10}$.

8 Boecio, In Categorias Aristotelis (PL, 64, 160 A): "Haec quidem est tempori introductionis, et simplicis expositionis apta sententia, quam nos nunc Porphyrium sequentes, quod videbatur expedior esse planiorque digessimus». Cf. LIBERA, A. de, «L'onto-théo-logique de Boèce: doctrine des catégories et théorie de la prédication dans le De trinitate», en: BRUUn U. y CoRTi L. (eds.), Les Catégories et leur histoire, Vrin, París 2005, 175-222 y EBBESEN, S., "The Aristotelian commentator» en: Marenbon, J. (ed.), The Cambridge Companion to Boethius, Cambridge University Press, Cambridge 2009, 45-46.

9 Boulnors, O., «Les catégories», op. cit., 358-359: «Si l'on soutient que la doctrine des Catégories porte sur les choses ou les genres de l'être, la doctrine des prédicables débouche sur un réalisme; si l'on soutient qu'elle porte sur les voces, les mots, on est conduit au vocalisme ou au nominalisme. C'est donc à même l'interprétation des Catégories que se décide une option pour les trois «traditions interprétatives», nominalisme, réalisme, conceptualisme». En este punto O. Boulnois sigue las observaciones de LiBERA, A. de, «Introduction» en: Porphyre, Isagoge, Vrin, París 1998, xii-Xvii y xl).

10 Avicena, Logyca, pars III (ed. de Venicia, 1508, 12ra): «Ponamus autem in hoc exemplum generis dicentes: quod animal est in se quodam: et idem est utrum sit sensibile: aut sit intellectum in anima. In se autem huius: nec est universale: nec est singulare. Si enim in se esset universale: ita quod animalitas ex hoc quod est animalitas est universalis: oporteret nullum animal esse singulare: sed omne animal esset universale. Si autem animal ex hoc quod est animal esset singulare: impossibile esset esse plus quam unum singulare, scilicet, ipsum singulare cui (?) animalitas; et 
Así consideradas, la universalidad es el modo propio de aprehensión de la naturaleza por el intelecto y la individuación es el modo propio de darse la naturaleza como entidad extramental. En el comentario a la Isagoge, siguiendo esta concepción aviceniana de la naturaleza y del universal, Escoto afirma:

Hay que decir que el universal es en sí inteligible. Eso es manifiesto así: el primer objeto del intelecto, a saber, el 'qué-es', es inteligido bajo la razón de universal. Sin embargo, esta razón no es esencialmente idéntica con el 'qué-es', sino que es un modo accidental suyo. Por ello, el intelecto puede conocer la diferencia entre su objeto primero y aquel modo, porque puede distinguir entre todas aquellas cosas que no son esencialmente idénticas. Sin embargo, toda virtud que por sí conoce la diferencia entre dos, conoce uno y otro extremo bajo su propia razón, como dice Aristóteles en el segundo libro Sobre el alma ${ }^{11}$. Y por esto prueba la existencia del sentido común. Por ello, el intelecto puede conocer aquel modo o razón universal en sí y bajo una razón propia. De este modo, reflexionando, el intelecto se conoce y conoce su operación y el modo de operar y otros que se encuentran en él ${ }^{12}$.

En esta solución, el universal es intencional: un concepto causado por el intelecto, gracias al cual la naturaleza es concebida bajo el modo de lo universal. Lo universal y lo concebido universalmente son esencialmente distintos.

La constitución de la unidad intencional de las categorías sobre el «concepto de categoría» obedece al mismo gesto de distinción que Escoto lleva a cabo en el comentario a la Isagoge, entre la esencia nuda y el modo intencional de su aprehensión por el intelecto. El concepto de categoría es el modo intencional bajo el cual el intelecto aprehende la diversidad categorial.

Con todo, el punto de partida sigue siendo la interpretación de Boecio. En efecto, según esta interpretación el tratado de las Categorías se ocupa de «las voces (voces) que significan las cosas en tanto que las significan ${ }^{13}$. Así considerado el objeto del que tratan las Categorías, el énfasis recae sobre la relación entre las palabras y las cosas (no sobre los conceptos). Por esta razón, se ha considerado, no

esset impossibile alius singulare esset animal. Animal autem in se est quodam intellectu in mente quod sit animal: et secundum hoc quod intelligitur esse animal non est nisi animal tantum. Si autem praeter hoc intelligitur esse universale: aut singulare: aut aliquid aliud : iam intelligitur praeter hoc quodam super id quod est animal quod accidit animalitati». Cfr. también AvicenNA, Philosophia prima sive scientia divina, V, 1 (AvL, II, 228 ss.).

11 Aristote, De Anima, II (B), 2, 426b 15-23 (ed. R.-A. Gauthier, 182).

12 Duns Scotus, In Porph., q. 5, n. 4 (OPh, I, 27-28): «Dicendum quod universale est per se intelligibile. Quod patet sic: primum obiectum intellectus, scilicet 'quod quid est' intelligitur sub ratione universalis. Illa autem ratio non est idem essentialiter cum illo 'quod quid est', sed modus eius accidentalis. Igitur intellectus potest cognoscere differentiam inter suum primum obiectum et illum modum, quia potest distinguere inter omnia quae non sunt essentialiter eadem. Sed omnis virtus cognoscens per se differentiam inter aliqua duo, cognoscit utrumque extremum sub propria ratione, per Aristotelem II De anima. Per hoc enim probat sensum communem esse. Igitur intellectus potest cognoscere illum modum sive rationem universalis per se et sub propria ratione. Hoc modo, reflectendo, cognoscit intellectus se et suam operationem et modum operandi et cetera que sibi insunt».

${ }_{13}$ Boecio, In Categorias Aristotelis (PL, 64, 160 AB): «Est igitur hujus operis intentio de vocibus res significantibus, in eo quod significantes sunt pertractare. (...) Sed nunc ad propositum revertamur, sitque in praesens praedicamentorum intentio, quae superius est comprehensa, id est, de primis vocibus significantibus prima rerum genera, in eo quod significantes sunt disputare». 
sin fundamento, que se trata de una posición que defiende una aproximación lingüística a las categorías ${ }^{14}$. Para Boecio la consideración de las categorías no pasa ni por los conceptos o intenciones lógicas que intervienen en su tratamiento, ni por las cosas en tanto tales en su división más general. Robert Kilwardby, por ejemplo, se expresa en términos semejantes y se remite expresamente a la lectura boeciana: «En efecto, como dice Boecio, la ciencia de las Categorías se ocupa de las diez voces que significan los diez primeros géneros de las cosas (...) se ocupa de las voces en tanto que son significantes» ${ }^{15}$.

\section{El horizonte intenCIONAL DEL TRATADO DE LAS CATEGORÍAS}

Escoto abre su comentario preguntándose en la primera cuestión «si el libro de las Categorías se ocupa de las diez voces que significan los diez géneros de cosas» ${ }^{16}$, situando así su problemática sobre la tensión establecida por la tradición porfirioboeciana. Sin embargo, contra esta tradición y en sintonía con Avicena, la respuesta de Escoto a esta pregunta distingue la dimensión lógica, anterior a la enunciación y relativa al orden intencional del pensamiento, de su contenido en el lenguaje ${ }^{17}$. Antes de las diez voces, ocurre aquello que es significado por ellas y que no necesita de ellas para tener su existencia mental. La respuesta a la pregunta por el objeto del tratado de las Categorías se dirige hacia la consideración de aquello que ellas tienen en común en la dimensión lógica o intencional, sus significados respectos, o mejor aún, el concepto mismo de significado:

Este libro no se ocupa de las diez voces como de su sujeto primero (de primo subiecto) - y ninguna parte de la lógica se ocupa de la voz, porque todas las

14 Cf. Ebbesen, S., «Les Catégories au Moyen Âge et au début de la modernité» en: BRuun U. y Corti L., op. cit., 257): «La question de savoir si les Catégories sont un ouvrage qui traite des mots ( $\varphi \omega v \alpha i ́)$, des choses ( $\pi \rho \alpha ́ \gamma \eta \alpha \tau \alpha)$, des concepts $(v o \eta ́ \mu \alpha \tau \alpha)$ ou d'une sorte de combinaison des trois a constitué un terrain d'affrontement célèbre entre les commentateurs anciens. Seules les deux premières alternatives (mots ou choses) ont été transmises aux médiévaux par Boèce, et avec elles la réponse 'nominaliste' de Porphyre —les mots dans leur capacité à être des signes des choses». Las cursivas son mías.

15 Kilwardby, R., Notulae super Librum Praedicamentorum, prooemium (ed. Conti): «Est igitur, ut dicit Boethius, scientia Praedicamentorum de $\mathrm{X}$ vocibus $\mathrm{X}$ prima rerum genera significantibus. Non enim est de vocibus penes diversas figurationes vocum quae sunt inflexio casuum aut temporum, set de voibus in quantum significativae sunt». Me honra poder agradecer aquí al profesor Alessandro Conti por su gentiliza en facilitarnos el acceso a una versión preliminar de la transcripción de su futura edición de este texto de Kilwardby y la profesor Olivier Boulnois por su gestión con el profesor Conti. Para una visión de conjunto de esta tradición en el siglo XIII, cfr. PINI, G., Categories and Logic in Duns Scotus: An Interpretation of Aristotle's Categories in the Late Thirteenth Century, Brill, Leiden-Boston-Colonia 2002; Biard, J. \& Rosier-Catach, I. (eds.), La tradition médiévale des catégories (XIIe-XVe siècles), Éditions de l'Institut Supérieur de Philosophie y Éditions Peeters, Louvain-la-Neuve Louvain-París-Dudley (MA) 2003; BRUUn, O. y CoRTI, L. op. cit.; Newton, Ll. A. (ed.), Medieval commentaries on Aristotle's Categories, Brill, Leiden-Boston, 2008 y, del mismo autor, Ll. A., «Introduction», en: Duns Scotus, J., Questions on Aristotle's Categories, The Catholic University of America Press, Washington 2014, 3-37.

16 Duns Scotus, In Praed., q. 1 (OPh, I, 249).

17 Cf. Salinas, H. H., "Observaciones sobre la constitución medieval de la lógica: de scientia sermocinalis a scientia rationalis», en: Universitas Philosophica, 64, 2015, 96-111. 
propiedades del silogismo y todas sus partes se hallan en él (sibi inesse) según el ser que tienen en la mente, aunque no sean proferidas, como es manifiesto inductivamente. Sin embargo, este libro [de las Categorías] se ocupa de algo anterior, que con respecto a la voz significante únicamente tiene la razón de significado ${ }^{18}$.

Con esta distinción entre el orden lógico intencional y el orden lingüístico, Escoto subraya la importancia de los significados categoriales, es decir, de los conceptos que cada una de las categorías significa y, para ser precisos, de su razón común, el concepto mismo de significado, como objeto propio del tratado. Para Escoto, el tratado aristotélico se ocupa de la dimensión lógica de las categorías, cuyo elemento común es el concepto de significado. Esta respuesta será precisada por Escoto, con el fin de evitar una confusión que viera en las categorías, consideradas desde el punto de vista lógico, o bien nombres o cosas significadas por nombres.

Escoto evita así dos lecturas a su juicio erróneas: una que inclinaría el tratado hacia la gramática (las categorías son nombres) y otra que lo inclinaría hacia la metafísica (las categorías corresponden a las divisiones más generales del ser). Recordemos que en su comentario a la Isagoge, Escoto estableció que el tratado de las Categorías hace parte de los tratados lógicos de Aristóteles y que por lo tanto se ocupa de una parte del objeto de la lógica, aquella que trata de los conceptos simples formados por el intelecto ${ }^{19}$.

\section{El PROBLEMA DEL SIGNIFICADO}

Sobre ese telón de fondo deben ser entendidas las siguientes afirmaciones de Escoto, algo desconcertantes si se toman al pie de la letra:

Por lo tanto, según esto hay que poner como sujeto primero de la lógica la voz. significante. Y así, el sujeto primero de este libro «Las Categorías» es la voz que significa el concepto simple (vox significans conceptum simplicem), y en grado sumo, las diez voces que significan los diez géneros porque todas a estas diez se reducen todas las otras voces ${ }^{20}$.

18 Duns Scotus, In Praed., q. 1 n. 11 (OPh, I, 251): «Iste liber non est de decem vocibus ut de primo subiecto - nec aliqua pars logicae est de voce, quia omnes passiones syllogismi et omnium partium eius posunt sibi inesse secundum esse quod habent in mente etsi non proferantur, ut patet inductive-, sed est de aliquo priore, quod respectu vocis signifcativae tantum habet rationem significati». (trad. Salinas, 280). Las cursivas son mías.

19 Duns Scotus, In Porph., q. 3 n. 7 (OPh, I, 12-13): «Dicitur quod subiectum logicae est conceptus formatus ab actu rationis, quia ille communis est omnibus consideratis in logica. Nam cum actus rationis sit triplex: primus indivisibilium intelligentia, secundus compositio vel divisio illorum indivisibilium, tertius discursus a noto ad ignotum: de conceptu formato a primo actu rationis est liber Praedicamentorum, qui est de incomplexo; de conceptu formato a secundo actu est liber Perihermenias, qui est de enuntiatione; de conceptu formato per tertium actum est nova logica quae est de syllogismo et eius partibus subiectivis». Aunque el objeto propio de la lógica es el silogismo, Escoto reivindica el derecho de las segundas intenciones (conceptos o entia rationis) a jugar un papel de objeto común de la lógica. Y en la división de las partes de la lógica, el tratado de las Catégories se ocupa de los conceptos simples formados por el intelecto.

20 Duns Scotus, In Praed., q. 1 n. 21 (OPh, I, 255): «Secundum hoc igitur primum subiectum logicaes est ponendum vox significativa. Et sic subiectum primum huius libri est vox significans conceptum simplicem, et maxime decem voces significantes decem genera, quia ad illas omnes aliae reducuntur». (trad. Salinas, 286). 
Aunque esta no es una respuesta errónea, no es la respuesta definitiva de Escoto. A primera vista, la tesis según la cual el objeto de la lógica es la voz significante parece oponerse a la tesis tradicional de Escoto según la cual el objeto propio de la lógica es el silogismo ${ }^{21}$. Sin embargo, en el contexto de este pasaje, la voz significante sí puede ser el objeto de la lógica, bajo la condición de considerarla en la perspectiva allí señalada, en tanto signo de un concepto. La continuación del pasaje radicaliza la respuesta, pues si la voz significante es el objeto del tratado de las Categorías en tanto signo de un concepto simple, "las diez voces que significan los diez géneros» lo son en grado sumo. Distinguimos pues (a) la voz que significa un concepto en tanto objeto de la lógica, (b) la voz que significa un concepto simple como objeto de las Categorías y (c) las diez voces que significan los diez géneros, entendidos como los diez conceptos más generales, en tanto objeto propio del tratado de las Categorías y en tanto toda voz se reduce a una de estas diez voces.

Pero, ¿cómo interpretar esta última afirmación vista desde la teoría del significado de Escoto? Hay que recordar que Escoto estudia el problema del significado en sus dos comentarios al De Interpretatione de Aristóteles ${ }^{22}$. Como elemento común a sus distintos tratamientos del problema, se mantiene la tesis según la cual un nombre significa una cosa, pero no en tanto realidad extramental, sino en tanto cosa concebida ${ }^{23}$.

Teniendo esto en mente, creemos que en el comentario a las Categorías, Escoto utiliza el lenguaje del «concepto» para expresar lo que su teoría del significado llama la cosa en tanto concebida (res ut concipitur o res ut intelligitur), pues cualquier voz que significa un concepto es reductible a las categorías comprendidas como las diez voces que significan los diez géneros más generales de las cosas. En esta formulación los diez géneros de las cosas se identifican con los diez conceptos más simples. Se da un tránsito entre voz, cosa y concepto en el que lo significado es la cosa en tanto concebida o la cosa en su concepto. Es por esto que los diez géneros pueden identificarse con los diez conceptos más simples. De este modo, el concepto se identifica con la cosa en su existencia intencional, o en otros términos, con la

21 Duns Scotus, In Porph., q. 3 n. 20 (OPh, I, 16-17).

22 Duns Scotus, In Peri herm. I, 2, (OPh, II, 47-59): Utrum nomen significet rem vel speciem in amina y In Peri herm. II, 1, (OPh, II, 137-145): An nomen significet rem an passionem. Otros dos lugares donde se encuentra una solución madura al problema son la Ordinatio I, 27, 1-3, n. 83, (Vat. VI, 97) y la Lectura I, 27, 1-3, n. 51, (Vat. XVII, 357). Cfr. Perler, D., «Duns Scotus on Signification», en: Medieval Philosophy and Theology, 3, 1993, 97-120 y «Duns Scotus's Philosophy of Language», en: Williams, T. (ed.), The Cambridge Companion to Duns Scotus, Cambridge University Press, Cambridge 2003, 161-192; PINI, G., "Species, Concept, and Thing: Theories of Signification in the Second Half of the Thirteenth Century», en: Medieval Philosophy and Theology, 8, 1999, 21-52 y «Signification of names in Duns Scotus and Some of His Contemporaries», en: Vivarium, 39 (1), 2001, 20-51; Sondag, G., "Introduction», en: Duns Scot, J., Signification et vérité: Questions sur le Peri hermeneias d'Aristote, Vrin, París 2009, 7-60 y Salinas, H. H., "El problema del nombre común en Juan Duns Escoto», en: Universitas Philosophica, 56, 2011, 201-240.

23 Por ejemplo, Duns Scotus, In Peri herm. I, 2, n. 39, (OPh, II, 56): «Ad partem oppositam questionis dicitur quod res primo significatur, non tamen secundum quod exsistit quia nec sic intelligitur, sed secundum quod per se concipitur ab intellectu, hoc est ipsa essentia rei quae significatur per definitiones quae est primum obiectum intellectus. Ad omnes auctoritates in contrarium dicitur quod per speciem vel passionem vel conceptum vel quodcumque aliud in aliis auctoritatibus significatur 'res ut intelligitur', ad denotandum quod 'res ut existit' non significatur». 
cosa en tanto concebida, o incluso con la razón de concepto que comparten todas las cosas bajo la consideración que la ciencia lógica hace de las categorías ${ }^{24}$.

Es por esta razón, nos dice Escoto, que el tratado de las Categorías tiene por objeto "las diez voces que significan los diez géneros», porque todas las otras voces significantes están contenidas en las diez categorías y estas voces que son las categorías significan los conceptos o las cosas más generales en tanto concebidas. Subrayemos además ese movimiento por el cual Escoto pasa del análisis de cualquier voz significante a la reflexión sobre las diez voces significantes que son las categorías. No podemos pues olvidar que las categorías son estudiadas por la lógica en tanto conceptos simples que tienen en común la razón de «significado». En otros términos, las categorías, a pesar de su diversidad real, tienen un elemento común, no real, sino intencional, su carácter de significado.

De la problemática general del significado, se pasa a la problemática del significado de las categorías insistiendo sobre el carácter conceptual o lógico del significado. De este modo, Escoto se asegura de mostrar que la aproximación a las categorías en el tratado de las Categorías es lógica y no ontológica. Sin embargo, en cuanto a esta última afirmación, tenemos que hacer una distinción suplementaria para explicitar con mayor precisión la dimensión lógica del tratado.

\section{La diversidad categorial y el objeto del tratado de las Categorías}

Una vez delimitado el alcance de la dimensión lingüística de las categorías (en tanto son voces) y subordinado a su dimensión lógico-semántica, Escoto se pregunta en la cuestión 2, teniendo en cuenta el doble estatuto de las categorías en tanto cosas y conceptos, si ellas son el objeto tratado en el libro de las Categorías.

La respuesta de Escoto se funda en la distinción entre dos tratamientos de las categorías: «de un modo, en tanto son entes, y de otro, en tanto son consideradas por la razón o en tanto alguna propiedad causada por el intelecto se les atribuye» ${ }^{25}$. El primero es el enfoque propio del metafísico y el segundo el enfoque propio del lógico y, por lo tanto, el enfoque propio del tratado de las Categorías.

La lógica se ocupa de las categorías consideradas como los conceptos más generales que incluyen en sí sus propiedades lógicas, es decir, sus "predicados intencionales». Para el caso de la sustancia, por ejemplo, se consideran propiedades lógicas «ser predicado unívocamente» o «no estar en un sujeto». Y vistas en conjunto, se consideran propiedades lógicas de las categorías, por ejemplo «dividirse en especies», "tener muchas especies subalternadas» o "no tener un género superior (supraveniens)» ${ }^{26}$.

24 Sobre la lógica como ciencia en Escoto, ver SALINAS, H. H., «La determinación de la lógica como ciencia común intencional en Duns Escoto», en: Franciscanum, LVIII (165), 2016, 51-86.

25 Duns Scotus, In Praed., q. 2 n. 5 (OPh, I, 258): «Decem praedicamenta possunt dupliciter considerari: uno modo in quantum sunt entia; alio modo in quantum considerantur a ratione, sive in quantum aliqua proprietas causata ab intellectu eis attribuitur». (trad. Salinas, 289). Cfr. PINI, G., Categories and Logic, op. cit., 139 y Newton, Ll. A., «Duns Scotus's Account of a Propter Quid Science of the Categories», en: Newton, Ll. A. (ed.), Medieval commentaries, op. cit., 254.

26 Duns Scotus, In Praed., q. 2 n. 7 (OPh, I, 259). (trad. Salinas, 289-290). 
Sin embargo, el problema fundamental que se enfrenta para considerar las categorías como el objeto del tratado de las Categorías sigue siendo el problema de la unidad del objeto: ¿hay acaso un elemento común a las diez categorías para fundar sobre él, como sobre su objeto, la unidad de ese saber? La diversidad de las categorías exige una explicación suplementaria con el fin de poder fundar una ciencia lógica de las categorías sobre un objeto unívoco. En este contexto de los comentarios a los distintos libros del Órganon la pregunta por la unidad de las categorías recibe una reformulación de fondo: ¿En tanto que conceptos, tienen las categorías una unidad lógica, así como en tanto que entes, tienen una unidad de analogía fundada sobre la unidad de la substancia? A partir del contraste entre la aproximación metafísica y la aproximación lógica a las categorías, Escoto formula la pregunta por la unidad lógica de las categorías:

Sin embargo, puesto que estas diez < categorías > no son el sujeto (subiectum) de una única ciencia real, a saber de la metafísica, sino en cuanto hay en ellas un principio al cual las otras son atribuídas, como se encuentra en la Metafísica IV $^{27}$ y, en cuanto son consideradas por la razón, no hay ningún principio al cual sean atribuídas, ¿cómo constituyen un único sujeto ${ }^{28}$ ?

El problema es pues que la unidad metafísica de las categorías reposa sobre una atribución real de las otras categorías a la substancia, mientras que el enfoque lógico ignora esta dimensión real ${ }^{29}$. Escoto establece del siguiente modo la unidad lógica de las categorías:

Es necesario decir que es mayor la unidad de ellas en alguna propiedad causada por el intelecto que en cuanto son entes. Y así, puesto que esta ciencia no es una por una unidad de analogía, es necesario asignar algo intencional que sea común a ellas y sujeto primero (primum subiectum), puesto que el lógico considera por sí únicamente algo de tal tipo ${ }^{30}$.

En este pasaje encontramos, a propósito de las relaciones entre metafísica y lógica, un presupuesto central del análisis escotista del ente. En primer lugar, se consideran dos niveles diferenciados a cerca de la unidad del objeto. De un lado, la unidad de la metafísica como unidad de analogía, fundada sobre la atribución a

27 Aristóteles, Metaphysica, IV (Г), 2, 1003b16-17 (AL, XXV², 61): «Proprie vero primi est scientia, et ex quo alia pendent, et propter quod dicuntur». "Ahora bien, en todos los casos la ciencia se ocupa fundamentalmente de lo primero, es decir, de aquello de que las demás cosas dependen y en virtud de la cual reciben la denominación». (trad. Calvo Martínez, 164).

28 Duns Scotus, In Praed., q. 2 n. 8 (OPh, I, 259-260): «Sed cum ista decem non sint subiectum unius scientiae realis, scilicet metaphysicae, nisi in quantum est in istis unum primum ad quod alia attribuantur, ut habetur IV Metaphysicae; et in quantum considerantur a ratione nullum est ad quod attribuantur, quomodo sunt unum subiectum?». (trad. Salinas, 290).

${ }_{29}$ Hay que subrayar que cuando Escoto redacta este comentario, el objeto de la metafísica no es aún el ente en tanto que concepto unívoco (según la célebre solución escotista expuesta en la Ordinatio I, d. 3 qq. 1-3, Vat. III, 1-123), sino precisamente la substancia en tanto que categoría primera a la cual se atribuyen las otras categorías. Esta solución se encuentra en su comentario a la Metafísica, Duns Scotus, In Met., I, q. 1 nn. 91-109 (OPh, III, 46-53).

30 Duns Scotus, In Praed., q. 2 n. 9 (OPh, I, 260): «Oportet dicere quod maior est unitas eorum aliqua proprietate causata ab intellectu quam in quantum sunt entia. Et ita, cum haec scientia non sit una unitate analogiae, oportet asignare aliquid intentionale quod sit commune istis, et primum subiectum, quia de solo tali per se considerat logicus». (trad. Salinas, 290). 
la substancia de las otras categorías. Y de otro lado, la unidad de la lógica, unidad mayor que la de la metafísica, fundada sobre un rasgo universal de una propiedad lógica común a todas las categorías. Por lo demás, el pasaje anuncia de pasada, la solución de Escoto en la cuestión 4 de este comentario, a la pregunta sobre el estatuto semántico del término «ente»o «ser»: equívoco desde el punto de vista del lógico y análogo desde el punto de vista del metafísico.

Ahora bien, esta doble respuesta, que la consideración lógica de las categorías se ocupa de un rasgo intencional y la consideración metafísica de la substancia como elemento primero de atribución al cual las otras categorías son reconducidas, se apoya sobre el carácter intencional de la predicación lógica, es decir, sobre la causalidad del intelecto que produce las propiedades lógicas. Estas se encuentran por accidente en las categorías y en sí en el concepto intencional de «categoría». He aquí la respuesta de Escoto:

Por lo tanto, puede decirse que aquí <en el libro de las Categorías > se consideran las diez categorías en cuanto se atribuye a ellas algo causado por la razón, porque de otro modo no pueden ser consideradas por el lógico. Y de este modo, no solo tienen una unidad de analogía, sino también una unidad de univocación. Y esto unívoco en ellas, de este modo, es algo intencional, que es aquí el sujeto primero (primum subiectum). Esto puede llamarse «categoría» o «generalísimo», puesto que todas las propiedades que aquí se determinan por sí sobre esta diez, se determinan en cuanto ellas tiene la razón de "generalísimo» o de «categoría» 31 .

Este rasgo intencional común y unívoco a las diez categorías permite precisamente distinguir los dos tipos de unidad propios al modo metafísico y al modo lógico de tratar las categorías. De un lado (a) la unidad real de analogía de la metafísica, gracias a la atribución a la substancia del resto de las categorías, y (b) la unidad de univocidad lógica fundada sobre la razón o el concepto de «categoría» o de «lo más general».

Con esta segunda unidad se alcanza el grado máximo de unidad lógica que recubre tanto las categorías como sus propiedades lógicas. Así, esta unidad de univocidad, la univocidad que corresponde al concepto lógico «categoría», funda el tratamiento lógico-científico de las categorías cuyo objeto primero es este rasgo absolutamente general causado por el intelecto. Precisamente el tratamiento lógico y la pregunta sobre la cientificidad del tratado de las categorías permiten a Escoto construir una reflexión sobre el concepto intencional de "categoría en general», pues, por su parte, la aproximación metafísica a las categorías se mantiene anclada en la multiplicidad categorial y en la atribución de las otras categorías a la substancia. Por esta razón, desde el punto de vista metafísico la diversidad categorial tiene mayor relevancia que la unidad lógico-intencional del concepto de «categoría».

31 Duns Scotus, In Praed., q. 2 n. 19 (OPh, I, 262): «Ideo dici potest quod hic consideratur de decem praedicamentis in quantum eis attribuitur aliquid causatum a ratione, quia aliter non possunt considerari a logico. Et illo modo non habent tantum unitatem analogiae, sed etiam univocationis; et illud univocum istis illo modo est aliquod intentionale, quod est hic primum subjectum. Illud potest nominari 'praedicamentum' vel 'generalissimum', quia omnes proprietates quae per se determinantur hic de istis, determinantur de eis in quantum habent rationem generalissimi vel praedicamenti». (trad. Salinas, 293). Cfr. PINI, G., Categories and Logic, op. cit., 162. 
Sin embargo, subrayemos una vez más que el análisis lógico pertenece a un orden accidental con respecto al análisis metafísico. Duns Escoto lo acepta sin vacilación cuando presenta el objeto de la ciencia lógica de las categorías como el concepto intencional más general. En efecto, según una objeción totalmente asumida por Escoto, la determinación lógica de las categorías es per accidens porque se construye «únicamente en tanto algo se halla en ellas por accidente, a saber, algo intencional, porque nada de tal índole puede hallarse en ellas por sí ${ }^{32}$. Lejos de escapar a este reproche, por el contrario, asumiéndolo, Escoto nos presenta otro rasgo de la distinción esbozada aquí entre metafísica y lógica: «digo que no es inconveniente que el lógico únicamente considere las categorías por accidente (per accidens), pues el metafísico las considera por sí (per se)» ${ }^{33}$. Encontramos pues una suerte de compensación (o de proporcionalidad inversa con respecto a la metafísica) entre el grado de unidad adquirido por la lógica (unidad de univocidad) y el grado de proximidad lógica a las categorías en tanto entes (en la medida en que el tratamiento lógico solo es un tratamiento accidental). Lo intencional posee mayor unidad, pero es accidental con respecto a las categorías en sí, de las que no hace parte esencialmente, precisamente porque es causado por el intelecto y no hace parte de la definición de cada categoría. La lógica es una ciencia más segura, pero es también, con respecto a las categorías, una ciencia per accidens.

En este mismo sentido, puede decirse que este rasgo intencional es «causa» de las categorías, aunque no tengan una causa desde el punto de vista metafísico, en tanto las categorías son absolutamente primeras ${ }^{34}$. Así, Escoto afirma que «respecto de sus propiedades las categorías tienen, no obstante, una causa; principalmente con respecto a las propiedades intencionales que les pertenen en cuanto son consideradas por la razón $»^{35}$. Si desde el punto de vista metafísico nada es anterior a las categorías, es decir, en tanto se las trata por sí, cuando se las considera intencionalmente, sí hay algo anterior. La univocidad lógica se funda pues gracias a la causalidad del intelecto. Sin embargo, esta univocidad no elimina la analogía metafísica, pues cada una se sitúa en un orden científico diferente.

Sobre este fundamento, Escoto responde a la pregunta de la cuestión 3, ¿algún predicado intencional es unívoco a las diez categorías ${ }^{36}$ ? La respuesta distingue entre la diversidad real de las cosas y su unidad causada por el intelecto. Esta unidad le permite al intelecto concebir lo real de modo unificado, a pesar de su diversidad real, gracias a un único modo de concebirlas:

32 Duns Scotus, In Praed., q. 2 n. 20 (OPh, I, 262-263): «De substantia, quantitate etc. Tantum determinatur hic per accidens, quia tantummodo secundum aliquid quod inest eis per accidens, scilicet aliquod intentionale, quia nullum tale potest eis inesse per se». (trad. Salinas, 293).

33 Duns Scotus, In Praed., q. 2 n. 24 (OPh, I, 263): «Dico quod non est inconveniens illa tantum per accidens considerari a logico quae metaphysicus per se considerat». (trad. Salinas, 294).

34 Duns Scotus, In Praed., q. 2 n. 1 (OPh, I, 257): «Istis nihil est prius naturaliter cum sint prima». (trad. Salinas, 288).

35 Duns Scotus, In Praed., q. 2 n. 28 (OPh, I, 264): «Licet illorum in se non sit aliqua causa, tamen respectu suarum passionum habent causam, praecipue respectu passionum intentionalium quae insunt eis in quantum considerantur a ratione». (trad. Salinas, 295).

36 Duns Scotus, In Praed., q. 3 (OPh, I, 267): "An aliquod praedicatum intentionale sit univocum istis generalissimis». 
...algo intencional unívoco puede aplicarse a las cosas de todos los géneros, porque toda diversidad en las cosas de primera intención, entre ellas, no impide que ellas puedan concebirse por el intelecto según el mismo modo de concebir (modum concipiendi). Las intenciones se atribuyen a las categorías en cuanto son concebidas por el intelecto, y por ello las intenciones de la misma especie pueden atribuirse a diversas cosas ${ }^{37}$.

La univocidad de las intenciones descansa sobre su modo de ser concebidas y no implica ninguna univocidad a nivel de las cosas. Esto quiere decir que existe un plano donde una unidad o una distinción conceptual no corresponde a ninguna unidad real: el plano lógico. Al mismo tiempo, como lo mencionamos al comienzo de este estudio, esta solución se articula con los análisis de Escoto en su comentario a la Isagoge de Porfirio, a propósito de la naturaleza del universal lógico definido como el modo de aprehensión intelectual de la naturaleza o esencia de algo. Aquí, la diversidad categorial es aprehendida por un modo de concebir propio del intelecto que unifica las categorías al concebirlas bajo la razón de «categoría», dejando intacta su diversidad real.

Así, predicar este rasgo intencional (el concepto de categoría), en tanto concepto de segunda intención, de una categoría en tanto concepto de primera intención, configura esta forma de predicación que Escoto llama la predicación denominati$\mathrm{va}^{38}$, en la que el predicado es el modus bajo el cual el intelecto aprehende el concepto del sujeto. Se trata de un tipo de predicación accidental en la medida en que el concepto de segunda intención no está incluido en la definición del concepto de primera intención. Es el caso de la proposición, «la substancia es una categoría», pues la substancia en tanto substancia no incluye dentro de su definición el concepto de categoría, pues de lo contrario, la substancia no sería una categoría, es decir, un género generalísimo, sino una especie del género categoría.

Esta particularidad da lugar a una objeción contra la univocidad del concepto de categoría. El predicado intencional, en este caso el concepto de «categoría», no

37 Duns Scotus, In Praed., q. 3 n. 8 (OPh, I, 269): «Aliquod intentionale univocum potest applicari rebus omniun generum, quia omnis diversitas in rebus primae intentionis inter se non impedit ipsas posse concipi ab intellectu per eundem modum concipiendi. Intentiones autem eis attribuuntur in quantum ab intellectu concipiuntur, et ideo intentiones eaedem specie possunt diversis rebus attribui». (trad. Salinas, 298). Cfr. PINI, G., Categories and Logic, op. cit., 143.

38 Este tipo de predicación pertenece efectivamente al orden de la predicación accidental, como lo recuerdan Libera A. de \& Michon, C., "Glossaire de sources: les origines du vocabulaire médiéval de l'ontologie», en: Thomas d'Aouin y Dietrich de Freiberg. L'Être et l'essence. Le vocabulaire médiéval de l'ontologie: Deux traités De ente et essentia de Thomas d'Aquin et Dietrich de Freiberg, Seuil, París 1996, 17, n. 1: "la prédication accidentelle se fait en disant de la substance un terme paronyme («blanc») du nom de l'accident («blancheur»), qui est dans la substance ( la blancheur est dans Socrate»). Les médiévaux parleront de "prédication dénominative», en raison de l'usage d'un terme («blanc») dont la signification est double, puisqu'il signifie l'accident et la substance, ou le composé - accidentel- de deux». En ese sentido la predicación accidental es llamada por los medievales "predicación denominativa». Sin embargo, en el caso preciso del empleo escotista de esta expresión, no se trata solamente de una predicación accidental, sino de una prédicación accidental en el orden lógico, en la que al menos el predicado de la proposición pertenece al orden intencional. Cf. LiBERA, A. de, «Prédication» en: CAssin, B. (dir.), Vocabulaire européen des philosophies: Dictionnaire des intraduisibles, Le Robert-Seuil, París 2004, 1008-1016. 
sería unívoco a las categorías, precisamente porque no sería predicable de ellas esencialmente, in quid ${ }^{39}$.

La respuesta de Duns Escoto descansa sobre una distinción entre dos tipos de univocidad. De un lado, una univocidad según la cual «algo unívoco se predica de muchos» (aliquid univocum praedicari de multis) y del otro, una univocidad según la cual «hay un predicado unívoco de muchos» (esse univocum praedicatum de multis). El predicado intencional «categoría», en tanto modus concipiendi (n. 8), únicamente es unívoco según el primer modo, bajo la forma de la predicación denominativa, pues la univocidad de acuerdo al segundo modo corresponde a predicados esenciales o in quid. En ese sentido, Escoto asume la objeción en la medida en que predicar el concepto intencional unívoco de «categoría» de las diez categorías no corresponde a un rasgo esencial de cada categoría. Sin embargo, este concepto intencional puede ser predicado de las categorías, siendo unívoco, de manera no unívoca, aceptando la diversidad categorial:

Es distinto decir «algo unívoco predicado de muchos» y «hay un predicado unívoco de muchos». El denominativo es unívoco en el primer modo, no en el segundo modo, como muestra la razón. Es por ello que concedo la conclusión (n. 4) según la cual ningún predicado intencional es un predicado unívoco de las cosas de primera intención, pero algo intencional unívoco en sí se predica de estas no unívocamente ${ }^{40}$.

La última negación debe ser leída, en nuestra opinión, como un acento sobre la distinción entre el primer tipo de univocidad, propia del concepto de segunda intención, y el segundo tipo de univocidad propio de un rasgo esencial predicado de un sujeto. Comprendido de ese modo, el predicado intencional no es unívoco de acuerdo al segundo modo. El sentido de la respuesta escotista, al menos para nosotros, parece claro: la univocidad intencional tiene que ver con el modo de concebir (modus concipiendi) las categorías, unívoco en sí, y no con la naturaleza de las categorías significadas en la predicación. Esta distinción se explica gracias al modelo de la predicación denominativa. Podríamos pues distinguir entre la intención segunda unívoca y el ser real unívoco. En el caso de las intenciones, su univocidad no implica bajo ninguna circunstancia la univocidad real de las cosas. Con esta distinción, Escoto emprenderá en la siguiente cuestión, la refutación de la univocidad y de la analogía lógica del término «ser», para sostener su equivocidad lógica. Como dice al final de la cuestión: «sed de hoc magis dicetur postea» ${ }^{41}$.

39 Duns Scotus, In Praed., q. 3, n. 4 (OPh, I, 267-268): «Item, nullum univocum est denominativum; sed praedicatum intentionale tantum dicitur, de illis quae sunt res primae intentionis, denominative; ergo nullum praedicatum intentionale est univocum istis». (trad. Salinas, 295).

40 Duns Scotus, In Praed., q. 3 n. 14 (OPh, I, 271): «Aliud est dicere 'aliquid univocum praedicari de multis', et 'esse univocum praedicatum de multis'. Denominativum primo modo est univocum, secundo modo non, ut ostendit ratio. Unde concedo conclusionem quod nullum praedicatum intentionale est univocum praedicatum de rebus primae intentionis, sed aliquod intentionale univocum in se praedicatur de istis non univoce». (trad. Salinas, 299). Cf. Newton, Ll. A., «Duns Scotus's Account», op. cit., 255.

41 Duns Scotus, In Praed., q. 3 n. 16 (OPh, I, 277). 


\section{Conclusiones}

Hecho este recorrido, algunas constataciones se imponen. El orden intencional corresponde al espacio de las operaciones y objetos causados por el intelecto. En el caso de las categorías, Escoto distingue, de un lado, su dimensión real, y del otro, su modo de ser concebidas bajo el concepto mismo de "categoría». La dimensión real corresponde al ámbito de ocupación del metafísico y por ello la metafísica se funda sobre una unidad de analogía en la que a la substancia se le atribuyen las otras categorías. Como vemos, estamos muy lejos de una metafísica fundada en la unidad de univocidad del concepto de ente, como será el caso en la obra teológica de Escoto.

Puesto que la dimensión lógica, en la que se sitúa el concepto de «categoría», es exclusivamente intencional, Escoto puede mantener simultáneamente tanto la diversidad real de las categorías, limitada por su unidad de analogía, como su unidad lógica de univocidad, fundada en el concepto lógico de categoría.

Aunque esta distinción será permanente en el pensamiento de Escoto, y dará lugar a una autonomización del espacio lógico con respecto al lenguaje y a la ontología anclada en la producción intencional del mismo espacio lógico, lo diferencial en este período temprano de su pensamiento es la concepción de la metafísica bajo la analogía real fundada en la atribución de las otras categorías a la substancia y, por otro lado, su rechazo de la univocidad y la analogía lógicas del concepto de ser. Sin embargo, este es un tema que exigirá de nosotros, un desarrollo independiente en un estudio por venir.

\section{Bibliografía}

\section{Fuentes primarias}

ARistóteles, Metafísica, (trad. Tomás Calvo Martínez), Gredos, Madrid 1994.

Aristoteles Latinus, De Anima, ed. Gauthier, R.-A., en Tomás de Aquino, Sententia libri De Anima, ed. Gauthier, R.-A., en Opera omnia t. XLV.1, 2ª ed., Commissio Leonina-J. Vrin, Rome-Paris 1984.

Avicenna, Liber de philosophia prima sive Scientia divina I-IV, ed. VAN RIET, S., «Avicenna Latinus», Peeters-Brill, Louvain-Leyde 1977.

Avicena, Logyca, s. ed., Venetiis 1508, ff. 2-12.

Boecio, In Categorias Aristotelis libri quatuor, ed. Migne, J.-P., «Patrologia Latina, 64», París, 1847.

Duns Escoto, J., Opera omnia III: Ordinatio I, d. 3, ed. Balić, C. et al., Typis Polyglottis, Civitas Vaticana 1954.

-, Opera omnia VI: Ordinatio I, dd. 26-48, ed. Balić, C. et al., Typis Polyglottis, Civitas Vaticana 1963.

—, Opera omnia XVI: Lectura, Prologus - I, dd. 1-7, ed. Balić, C. et al., Typis Polyglottis, Civitas Vaticana 1960.

-, Opera philosophica I: Quaestiones In Librum Porphyrii Isagoge et Quaestiones Super Praedicamenta Aristotelis, eds. Andrews, R. et al., The Franciscan Institute, St. Bonaventure (NY) 1999. 
—, Opera philosophica II: Quaestiones in Libros Perihermenias Aristotelis, Quaestiones Super Librum Elenchorum Aristotelis, et Theoremata, eds. ANDREws, R. et al., The Franciscan Institute-The Catholic University of America, St. Bonaventure (NY)-Washington 2004.

-, Opera philosophica III-IV: Quaestiones super libros metaphysicorum Aristotelis, eds. ANDREws, R. et al., The Franciscan Institute, St. Bonaventure (NY) 1997.

Duns Scot, J., Sur la connaissance de Dieu et l'univocité de l'étant, (introducción, traducción francesa y comentario O. Boulnois), PUF, París 1988.

Duns Scot, J., Questions on Aristotle's Categories, (trad. inglesa Lloyd A. Newton), The Catholic University of America Press, Washington 2014.

Duns Escoto, J., Cuestiones sobre las Categorías de Aristóteles, qq. 1-3, (trad. castellana H. H. Salinas), en: Universitas Philosophica, 67, 2016, 275-302.

Porphyre, Isagoge. (Trad. francesa A. de Libera y A.-Ph. Segonds). Introducción y notas A. de Libera. Vrin, París 1998.

Kilwardby, R., Notulae super Librum Praedicamentorum, transcripción preliminar de A. Conti.

Bibliografía secundaria

Asztalos, M., Murdoch J. E. \& Ninniluoto, I. (eds.), Knowledge and the Sciences in Medieval Philosophy. Proceedings of The Eighth International Congress of Medieval Philosophy, Vol. 1, Acta Philosophica Fennica, Helsinki 1990.

Biard, J. \& Rosier-CATach, I. (eds.), La tradition médiévale des catégories (XIIe-XVe siècles), Éditions de l'Institut Supérieur de Philosophie y Éditions Peeters, Louvain-la-Neuve Louvain-París-Dudley (MA) 2003.

Boulnois, O., "La destruction de l'analogie et l'instauration de la métaphysique», en: Duns Sсот, J., Sur la connaissance de Dieu et l'univocité de l'étant, PUF, París 1988, 11-81.

Boulnors, O., «Analogie et univocité selon Duns Scot: La double destruction», en: Les Études philosophiques, «L'analogie», 3-4, 1989, 347-369.

Boulnois, O., "Les catégories selon Duns Scot», en: Carbajo NúñEz, M. (ed.), Giovanni Duns Scoto: Studi e ricerche nel VII Centenario della sua morte, Vol.1, Antonianum, Roma 2008, 357-377.

Bruun, O. y Corti, L. (eds.), Les Catégories et leur histoire, Vrin, París 2005.

Ebbesen, S., «Les Catégories au Moyen Âge et au début de la modernité» en: Bruun U. y CorTI L. (eds.), Les Catégories et leur histoire, Vrin, París 2005, 245-274.

Ebbesen, S., «The Aristotelian commentator» en: Marenbon J. (ed.), The Cambridge Companion to Boethius, Cambridge University Press, Cambridge 2009, 34-55.

HonNefelder, L., La métaphysique comme science transcendantale: entre le Moyen Âge et les Temps modernes, (trad. I. Mandrella, revisada por O. Boulnois, J. Greisch y Ph. Capelle). PUF, París 2002.

Libera, A. de, «Introduction» en: Porphyre, Isagoge, Vrin, París 1998, viI-cxliI.

Libera, A. de, «Prédication» en: Cassin, B. (dir.), Vocabulaire européen des philosophies: Dictionnaire des intraduisibles, Le Robert-Seuil, París 2004, 1008-1016.

LiBera, A. de, «L'onto-théo-logique de Boèce: doctrine des catégories et théorie de la prédication dans le De trinitate», en: BRuUn U. y CoRTI L. (eds.), Les Catégories et leur histoire, Vrin, París 2005, 175-222.

Libera A. de \& Michon, C., "Glossaire de sources: les origines du vocabulaire médiéval de l'ontologie», en: Thomas d’Aquin y Dietrich de Freiberg. L'Être et l'essence. Le vocabulaire médiéval de l'ontologie: Deux traités De ente et essentia de Thomas d'Aquin et Dietrich de Freiberg, Presentados y traducidos por A. de Libera y C. Michon, Seuil, París 1996, 15-36.

Marenbon J. (ed.), The Cambridge Companion to Boethius, Cambridge University Press, Cambridge 2009, 34-55.

Newton, Ll. A. (ed.), Medieval commentaries on Aristotle's Categories, Brill, Leiden-Boston, 2008. 
Newton, Ll. A., «Duns Scotus's Account of a Propter Quid Science of the Categories », en: Newton, Ll. A. (ed.), Medieval commentaries on Aristotle's Categories, Brill, Leiden-Boston, 2008, 221-258.

Newton, Ll. A., «Introduction», en: Duns Scotus, J., Questions on Aristotle's Categories, The Catholic University of America Press, Washington 2014, 3-37.

Pasnau, R., "Science and Certainty», en: Pasnau, R. \& Dyke, Ch. van (eds.), The Cambridge History of Medieval Philosophy, vol. I, Cambridge University Press, Cambridge 2010, 357-368.

Pasnau, R. \& Dyke, Ch. van (eds.), The Cambridge History of Medieval Philosophy, vol. I, Cambridge University Press, Cambridge 2010.

Perler, D., «Duns Scotus on Signification», en: Medieval Philosophy and Theology, 3, 1993, 97-120.

—, «Duns Scotus's Philosophy of Language», en: Williams, T. (ed.), The Cambridge Companion to Duns Scotus, Cambridge University Press, Cambridge 2003, 161-192.

PINI, G., "Species, Concept, and Thing: Theories of Signification in the Second Half of the Thirteenth Century», en: Medieval Philosophy and Theology, 8, 1999, 21-52.

-, «Signification of names in Duns Scotus and Some of His Contemporaries», en: Vivarium, 39 (1), 2001, 20-51.

-, Categories and Logic in Duns Scotus: An Interpretation of Aristotle's Categories in the Late Thirteenth Century, Brill, Leiden-Boston-Colonia 2002.

RiJK, L. M. de, «The Posterior Analytics in the Latin West», en: Asztalos, M., Murdoch J. E. \& NiIniluoto, I. (eds.), Knowledge and the Sciences in Medieval Philosophy. Proceedings of The Eighth International Congress of Medieval Philosophy, Vol. 1, Acta Philosophica Fennica, Helsinki 1990, 104-127.

SALINAS, H. H., «El problema del nombre común en Juan Duns Escoto», en: Universitas Philosophica, 56, 2011, 201-240.

SALINAS, H. H., "Observaciones sobre la constitución medieval de la lógica: de scientia sermocinalis a scientia rationalis», en: Universitas Philosophica, 64, 2015, 96-111.

SALINAS, H. H., «La determinación de la lógica como ciencia común intencional en Duns Esсото», en: Franciscanum, LVIII (165), 2016, 51-86.

Salinas, H. H., "Juan Duns Escoto: Cuestiones sobre las Categorías de Aristóteles, qq. 1-3», en: Universitas Philosophica, 67, 2016, 275-302.

Serene, E., «Demonstrative science», en Kretzmann, N., Kenny, A., \& Pinborg, J. (eds.), The Cambridge History of Later Medieval Philosophy, Cambridge University Press, Cambridge 1982, 496-517.

SondaG, G., «Introduction», en: Duns Scot, J., Signification et vérité: Questions sur le Peri hermeneias d'Aristote, Vrin, París 2009, 7-60.

Williams, T. (ed.), The Cambridge Companion to Duns Scotus, Cambridge University Press, Cambridge 2003.

Pontificia Universidad Javeriana

hsalinas@javeriana.edu.co

Héctor Hernando Salinas

[Artículo aprobado para publicación en diciembre de 2016] 